\title{
Comportamento vegetativo e produtivo da videira e composição da uva em São Joaquim, Santa Catarina
}

\author{
Marcelo Borghezan ${ }^{(1)}$, Olavo Gavioli(1), Fábio Antônio Pit ${ }^{(1)}$ e Aparecido Lima da Silva(1) \\ (1)Universidade Federal de Santa Catarina, Centro de Ciências Agrárias, Departamento de Fitotecnia, Rodovia Admar Gonzaga, no 1.346, \\ CEP 88040-900 Florianópolis, SC. E-mail: mborghezan@hotmail.com, olavogavioli@hotmail.com, pitnick2004@yahoo.com.br, alsilva@cca.ufsc.br
}

Resumo - O objetivo deste trabalho foi avaliar o comportamento vegetativo e produtivo das variedades de videira Cabernet Sauvignon, Merlot e Sauvignon Blanc, e a composição da uva em São Joaquim, Santa Catarina. Foram avaliadas plantas de um vinhedo comercial, cultivado em espaldeira, a $1.293 \mathrm{~m}$ de altitude, durante os ciclos fenológicos 2005/2006 e 2006/2007. As variáveis meteorológicas, a fenologia, o desenvolvimento do dossel e a composição da uva na colheita foram comparados entre os ciclos. As temperaturas mais amenas influenciaram o ciclo fenológico das variedades Cabernet Sauvignon, Merlot e Sauvignon Blanc, que é mais longo e tardio que nas outras regiões vitícolas do Brasil. Houve influência significativa da precipitação pluvial sobre a maturação da uva, no ciclo 2006/2007. Os índices de desenvolvimento indicam a necessidade de ajuste no manejo do dossel para um maior equilíbrio entre o crescimento vegetativo e a produção. As variedades Cabernet Sauvignon, Merlot e Sauvignon Blanc apresentam elevada qualidade da uva no momento da colheita, sendo adequadas ao cultivo em São Joaquim, SC.

Termos para indexação: Vitis vinifera, Cabernet Sauvignon, fenologia, maturação, Merlot, Sauvignon Blanc.

\section{Vegetative and productive behavior of grapevines and composition of grapes in São Joaquim, Santa Catarina, Brazil}

\begin{abstract}
The objective of this work was to assess the vegetative and productive behavior of the grapevine varieties Cabernet Sauvignon, Merlot and Sauvignon Blanc, and the composition of grapes in São Joaquim, Santa Catarina State, Brazil. Grapevines from a commercial vineyard, grown on vertical trellis, at 1,293-m altitude were evaluated during the 2005/2006 and 2006/2007 phenological cycles. Meteorological variables, phenology, canopy development, and composition of grapes at harvest were compared between cycles. Milder temperatures influenced the phenological cycle of the grapevine varieties Cabernet Sauvignon, Merlot and Sauvignon Blanc, which is longer and starts later than in the other wine growing regions of Brazil. Rainfall significantly influenced grape maturity in the 2006/2007 cycle. Development indexes indicate the need to adjust vine canopy management for a greater balance between vegetative growth and production. The grapevine varieties Cabernet Sauvignon, Merlot and Sauvignon Blanc show high quality of grapes at harvest, being suitable for cultivation in São Joaquim, Santa Catarina State, Brazil.
\end{abstract}

Index terms: Vitis vinifera, Cabernet Sauvignon, phenology, ripening, Merlot, Sauvignon Blanc.

\section{Introdução}

A viticultura de Santa Catarina tem se destacado recentemente frente aos demais stados produtores de vinhos finos no Brasil. Para diversos autores (Falcão et al., 2008; Silva et al., 2009; Miele et al., 2010), as uvas produzidas nas regiões de altitude acima de $900 \mathrm{~m}$ apresentam características próprias e distintas das cultivadas em outras áreas do país, além de maturação fenólica adequada à elaboração de vinhos.

As condições climáticas regionais possibilitaram o desenvolvimento de índices de classificação das regiões de cultivo da videira, que são agrupadas de acordo com seu ciclo fenológico, como proposto por Tonietto \& Carbonneau (2004). Segundo essa classificação, São Joaquim, SC, apresenta um clima vitícola "frio, de noites frias e úmido".

Em uma mesma região, os diferentes ciclos produtivos são fortemente influenciados pelas alterações microclimáticas, o que favorece a maturação da uva em estádios variados. Exemplos referentes às regiões clássicas de produção de uvas no mundo são encontrados nos trabalhos de Esteves \& Orgaz (2001), na região do Dão em Portugal; de Leeuwen et al. (2004),

Pesq. agropec. bras., Brasília, v.46, n.4, p.398-405, abr. 2011 
em Bordeaux na França; e de Zoecklein et al. (2008), na California, Estados Unidos. Essas variações provocam alterações no padrão de crescimento e desenvolvimento das plantas e afetam significativamente a composição da uva, dependendo do período do ciclo vegetativo em que ocorrem, o que resulta em safras com padrões de qualidade diferenciados (Jones \& Davis, 2000).

Sob condições controladas em casa de vegetação, Girona et al. (2009) observaram que quando o deficit hídrico ocorreu em uma fase anterior à mudança de coloração, a qualidade da uva foi afetada negativamente. Entretanto, quando essa privação ocorreu durante o período de maturação, houve melhoria da composição das bagas. Além da disponibilidade hídrica, outras variáveis, como temperatura, umidade relativa e radiação solar, podem afetar as plantas a campo (Leeuwen et al., 2004; Zoecklein et al., 2008).

O Planalto Serrano é uma região vitícola recente que apresenta características climáticas particulares, como: altitude superior a $900 \mathrm{~m}$, proximidade do Oceano Atlântico (cerca de $150 \mathrm{~km}$ ) e latitude de $28^{\circ} \mathrm{S}$. A determinação dos estádios fenológicos, dos índices de crescimento e produção, e das variações meteorológicas ao longo dos anos são informações necessárias para o desenvolvimento da viticultura.

O objetivo deste trabalho foi avaliar o comportamento vegetativo e produtivo das variedades de videira Cabernet Sauvignon, Merlot e Sauvignon Blanc, e a composição da uva em São Joaquim, SC, nos ciclos fenológicos 2005/2006 e 2006/2007.

\section{Material e Métodos}

As avaliações foram realizadas em um vinhedo comercial da empresa Villa Francioni Agro Negócios S.A., em São Joaquim, SC (28 $15^{\prime} 13^{\prime \prime}$ S, 49 $57^{\circ} 02^{\prime \prime} \mathrm{W}$, a $1.293 \mathrm{~m}$ de altitude). O plantio ocorreu em 2002. As plantas foram conduzidas em sistema espaldeira, espaçadas em 3,0 m entre linhas e $0,75 \mathrm{~m}$ entre plantas de Cabernet Sauvignon e Merlot e 1,0 m na Sauvignon Blanc, sendo enxertadas sobre o porta-enxerto 'Paulsen 1103', com orientação das filas norte-sul. $\mathrm{O}$ clima da região é classificado como $\mathrm{Cfb}$, segundo Köppen, e o solo como Cambissolo Húmico Alumínico (Santos et al., 2006).

As linhas foram protegidas por uma tela de polietileno antigranizo e antiUV, com sombreamento de 9\% (Lahuman Ltda., Sumaré, SP). A poda foi realizada em sistema de cordão esporonado unilateral, tendo-se deixado duas gemas por esporão. A carga de gemas foi definida pela empresa para limitar a produção, sendo mantidas cerca de 16 gemas por planta nas variedades tintas e 24 na Sauvignon Blanc, em ambos os ciclos fenológicos. A partir da brotação, os ramos foram conduzidos na posição vertical para uma melhor disposição do dossel. Foram avaliadas plantas sem desponte dos ramos, com brotações laterais (feminelas), tendo-se comparado os ciclos fenológicos 2005/2006 e 2006/2007. A desfolha na região dos cachos foi realizada durante a formação das bagas. As variedades encontravam-se no mesmo vinhedo, em filas vizinhas. Foram avaliadas 20 plantas por variedade, com 5 repetições e 4 plantas por parcela experimental.

As variáveis microclimáticas foram obtidas de uma estação meteorológica, localizada na estação experimental da Empresa de Pesquisa Agropecuária e Extensão Rural de Santa Catarina, a 1.415 m de altitude e $2.800 \mathrm{~m}$ de distância da área experimental. Os dados diários de precipitação e temperatura média, máxima e mínima foram cedidos pelo Centro de Informações de Recursos Ambientais e de Hidrometeorologia de Santa Catarina. A partir desses dados, foram calculados o índice de Winkler (soma térmica - ST) e o índice heliotérmico $(\mathrm{IH})$ de Huglin $\left({ }^{\circ} \mathrm{C}\right)$ (Blanco-Ward et al., 2007). A ST e o IH foram estimados pelas equações:

$$
\begin{gathered}
\mathrm{ST}=\sum\left[\left(\mathrm{T}_{\text {máxima }}+\mathrm{T}_{\text {mínima }}\right) / 2\right]-10, \mathrm{e} \\
\mathrm{IH}=\sum\left[\left(\mathrm{T}_{\text {máxima }}-10\right)+\left(\mathrm{T}_{\text {média }}-10\right)\right] / 2 \mathrm{k} .
\end{gathered}
$$

O coeficiente de correção $(k)$ foi considerado 1 (latitude $<40^{\circ}$ ), conforme descrito por Tonietto \& Carbonneau (2004). A ST (graus-dias - GD) e o IH foram estimados com temperatura-base de $10^{\circ} \mathrm{C}$ para a videira (Blanco-Ward et al., 2007). Ambos os índices foram calculados para o período entre os principais estádios fenológicos, tendo-se tomado como referência o ciclo da variedade Cabernet Sauvignon.

A fenologia foi avaliada segundo descrito por Leeuwen et al. (2004). Para a definição dos estádios fenológicos da videira, foi utilizada a escala $\mathrm{BBCH}$ (Lorenz et al., 1995). Foram descritos os estádios fenológicos: poda - data em que foi realizada a poda de inverno, estabelecida a partir da observação do início da brotação dos ramos; brotação - considerada quando $50 \%$ das gemas estavam no estádio de ponta verde (BBCH 07); floração - considerada quando 
$50 \%$ das flores estavam abertas $(\mathrm{BBCH} 65)$; início da mudança de cor - aparecimento das primeiras bagas com alteração de cor ou consistência (BBCH 81); mudança de cor ("véraison") - considerada quando $50 \%$ das bagas mudaram de coloração ou amoleceram (BBCH 85); colheita - estabelecida com base na avaliação da composição química das bagas; e queda total das folhas - considerada quando as plantas perderam 100\% das folhas (BBCH 97).

A produção foi avaliada durante a colheita a partir da pesagem dos cachos (kg por planta) de 20 plantas. O número de cachos e de ramos por planta foi avaliado mediante contagem em data pré-colheita, em 60 plantas. Com base nesses dados, estimou-se o número de cachos por ramo. A massa média dos cachos foi estimada a partir do número de cachos e da produção por planta. Já a massa média dos ramos foi avaliada no final do ciclo 2005/2006, no momento da poda, para o início do próximo ciclo, pela pesagem do material retirado (quilograma por planta) de 20 plantas. O índice de Ravaz foi estimado pela relação entre a produção e o peso da poda (ciclo 2005/2006). A área foliar foi estimada com as equações definidas por Borghezan et al. (2010), a partir da avaliação de todas as folhas de 15 ramos selecionados na parte central das plantas, no final da maturação das bagas, sendo que a área foliar das brotações laterais (feminelas) foi avaliada da mesma forma que as folhas do ramo principal. A área foliar total foi estimada pela área foliar de cada ramo, multiplicada pelo número médio de ramos por planta.

A colheita foi realizada quando o teor de sólidos solúveis totais foi superior a $23^{\circ} \mathrm{Brix}$, no ciclo 2005/2006, e acima de $21^{\circ}$ Brix, no ciclo 2006/2007. A composição da uva foi avaliada na colheita, tendo-se amostrado 200 bagas de forma aleatória em diferentes posições dos cachos. As bagas foram coletadas mediante corte do pedicelo, com tesoura, e as amostras foram acondicionadas em sacos plásticos e transportadas em caixas refrigeradas até o laboratório, em Florianópolis, SC. O mosto extraído de cada uma das três subamostras de 30 bagas foi analisado imediatamente, com base na metodologia descrita em Organization Internationale de la Vigne et du Vin (2009). Foram avaliados: teor de sólidos solúveis totais (SST), com um refratômetro digital com compensação de temperatura, modelo RTD 45 (Instrutherm, São Paulo, SP); acidez total titulável (ATT) por meio de titulação $(0,1 \mathrm{~N}$ de $\mathrm{NaOH})$ com indicador fenolftaleína (1\%); e pH avaliado em
aparelhoAD 1030, (ADWA, Szeged, Hungria) calibrado com soluções tampão pH 4,0 e pH 7,0. A coloração das sementes foi avaliada seguindo a metodologia proposta por Ristic \& Iland (2005), com 60 sementes para cada variedade. A mucilagem foi retirada e, em seguida, avaliou-se a coloração da face ventral e da face dorsal das sementes, em escala de coloração (Ristic \& Iland, 2005). A coloração foi definida pela média entre as duas faces das sementes.

Os dados de fenologia dos dois ciclos foram analisados em conjunto. Os estádios entre as variedades foram comparados pela análise de variância (ANOVA) e pelo teste de Tukey, a 5\% de probabilidade. Os dados de comportamento vegetativo e produtivo de cada ciclo foram analisados separadamente, com o programa Statistica versão 6.0, pelo teste t de Student, a 5\% de probabilidade (Steel et al., 1997).

\section{Resultados e Discussão}

A temperatura média diária foi semelhante entre os dois ciclos avaliados, com exceção do período final de maturação (Figura 1). Em setembro, a temperatura média se manteve em torno de $10^{\circ} \mathrm{C}$, tendo alcançado cerca de $20^{\circ} \mathrm{C}$ em meados de janeiro e decrescido para aproximadamente $12^{\circ} \mathrm{C}$ no final de abril. A temperatura mínima e máxima seguiram o mesmo padrão, com variação de aproximadamente $\pm 5^{\circ} \mathrm{C}$ em relação à média. A amplitude térmica (diferença entre a temperatura máxima e mínima do dia) foi de aproximadamente $10^{\circ} \mathrm{C}$.

O índice de Winkler foi superior em 135 GD, no ciclo 2006/2007, em comparação ao ciclo anterior (Tabela 1). Nos dois anos estudados, os valores foram inferiores ao definido como limite de clima vitícola

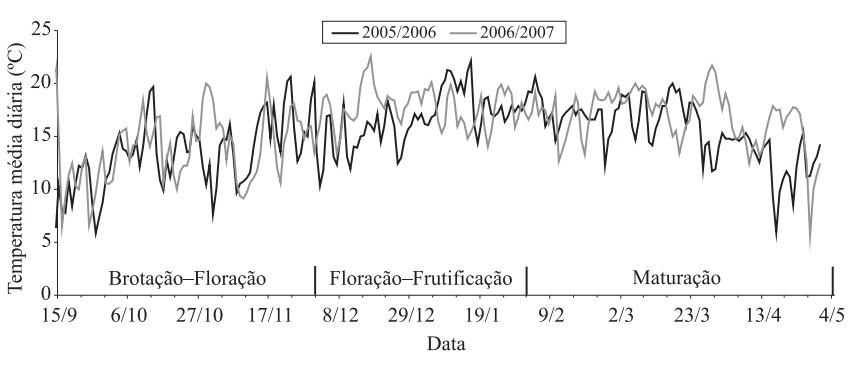

Figura 1. Temperatura média diária $\left({ }^{\circ} \mathrm{C}\right)$ durante o ciclo vegetativo da videira (Vitis vinifera L.) em São Joaquim, SC (15/9 a 30/4), nos ciclos 2005/2006 e 2006/2007. Os estádios fenológicos assinalados no eixo dos $\mathrm{x}$, estão baseados no ciclo da variedade Cabernet Sauvignon. 
frio por Winkler $(<1.371$ GD). O índice de Huglin (entre 1.500 e 1.800) também corrobora a classificação da região como de clima frio, conforme descrito por Tonietto \& Carbonneau (2004). Esses índices separam a região de São Joaquim, SC, das demais áreas de produção de uvas para elaboração de vinhos no Brasil, o que resulta em características diferenciadas dos vinhos, como verificado por Miele et al. (2010).

O volume de chuvas a partir de setembro (brotação) até o final de abril (colheita) no ciclo 2005/2006 foi inferior ao ciclo 2006/2007 (Tabela 1) e significativamente inferior à média histórica $(1.115 \mathrm{~mm})$ de precipitação para este período, em São Joaquim, SC. Quanto à precipitação pluvial durante o período de maturação, os ciclos diferiram significativamente entre si e em relação à média histórica, que é de cerca de $370 \mathrm{~mm}$ (Figura 2). No ciclo 2005/2006, o volume total foi de $147,9 \mathrm{~mm}$, sendo necessário irrigar as plantas a partir de meados de março. No ciclo seguinte, o excesso de dias chuvosos e o volume de chuvas acumulado $(501,9 \mathrm{~mm})$ favoreceram o desenvolvimento de doenças (podridão) que prejudicaram a qualidade da uva.

Os resultados obtidos no segundo ciclo de produção (2006/2007) diferem dos encontrados por outros autores, em anos anteriores, para a mesma região (Falcão et al., 2008), que verificaram menores índices pluviais durante os meses de maturação da uva de fevereiro a abril, o que refletiu em melhor qualidade da uva. Na Serra Gaúcha, região mais importante da vitivinicultura brasileira, em algumas safras, a uva é colhida em estádio menos avançado de maturação, principalmente em decorrência do excesso de precipitação, que favorece o desenvolvimento de doenças fúngicas nos cachos (Rizzon \& Miele, 2003). Historicamente, a precipitação pluvial não tem afetado negativamente a maturação da uva em São Joaquim, $\mathrm{SC}$, o que possibilita prolongar esse período e obter melhor qualidade no momento da colheita (Falcão et al., 2008; Silva et al., 2009).
Para todas as variedades, a brotação ocorreu aproximadamente uma semana após a poda (Figura 3). A variedade Sauvignon Blanc foi mais precoce, com aproximadamente 175 dias entre a brotação e a colheita. Já a Merlot, foi intermediária, com um ciclo em torno de 200 dias, embora não tenha diferido significativamente da variedade Cabernet Sauvignon, com um ciclo de 215 dias, em 2006/2007.

O ciclo de desenvolvimento vegetativo iniciou com a brotação, na segunda quinzena de setembro, e encerrou com a parada do crescimento dos ramos, na segunda quinzena de fevereiro, no início da maturação das bagas. Após esse período, os ramos iniciaram a lignificação, o ápice dos ramos e as brotações laterais cessaram o crescimento, e as folhas iniciaram a senescência. Esse comportamento foi descrito por Cloete et al. (2006), que afirmam que a redução do desenvolvimento dos ramos durante o início da maturação favorece a acumulação de compostos nas bagas, o que melhora a qualidade da uva.

O período de maturação iniciado após a mudança da cor e a alteração na consistência das bagas ("véraison") teve início em fevereiro, aproximadamente 70 dias após a plena floração (DAF), que ocorreu no final de novembro. A colheita da Sauvignon Blanc foi realizada em meados de março, aos 106 e 114 DAF nos ciclos 2005/2006 e 2006/2007, respectivamente. A variedade Merlot foi colhida aos 140 e $133 \mathrm{DAF}$, na primeira semana de abril, com período de maturação de 49 e 63 dias, respectivamente. A colheita da Cabernet Sauvignon foi realizada na segunda quinzena de abril, cerca de $150 \mathrm{DAF}$, após 10 e 11 semanas do início da maturação para os ciclos 2005/2006 e 2006/2007, respectivamente. O período de maturação das bagas apresentou variação entre as variedades (Figura 3), sendo menor para a Sauvignon Blanc (39 dias) do que para a Merlot (60 dias) e a Cabernet Sauvignon (76 dias).

Tabela 1. Precipitação pluvial e índices bioclimáticos (Winkler e Huglin) acumulados durante os estádios fenológicos da videira (Vitis vinifera L.) cultivada em São Joaquim, SC, nos ciclos 2005/2006 e 2006/2007.

\begin{tabular}{|c|c|c|c|c|c|c|}
\hline \multirow[t]{2}{*}{ Estádio fenológico } & \multicolumn{2}{|c|}{ Precipitação pluvial (mm) } & \multicolumn{2}{|c|}{ Soma térmica (GD) } & \multicolumn{2}{|c|}{ Índice heliotérmico (IH) } \\
\hline & $2005 / 2006$ & $2006 / 2007$ & $2005 / 2006$ & $2006 / 2007$ & $2005 / 2006$ & $2006 / 2007$ \\
\hline Brotação à floração & 378,9 & 304,9 & 276,6 & 227,8 & 450,3 & 395,8 \\
\hline Floração à maturação & 305,8 & 322,4 & 496,1 & 538,9 & 713,7 & 741,4 \\
\hline Maturação à colheita ${ }^{(1)}$ & 109,7 & 449,1 & 415,1 & 556,0 & 629,4 & 755,3 \\
\hline Brotação à colheita ${ }^{(1)}$ & 794,4 & $1.076,4$ & $1.187,8$ & $1.322,7$ & $1.793,4$ & $1.892,5$ \\
\hline
\end{tabular}

${ }^{(1)}$ Valores estimados com base no ciclo fenológico da variedade Cabernet Sauvignon. 
O ciclo fenológico da videira em São Joaquim, SC, é mais tardio em comparação às demais regiões vitícolas brasileiras. Rizzon \& Miele (2003) e Mota et al. (2008) encontraram ciclo médio de 150-160 dias para as variedades Merlot e Cabernet Sauvignon, cultivadas em Bento Gonçalves, RS. Esses resultados estão de acordo com as observações de Jones \& Davis (2000) em Bordeaux, ao relatarem ciclo de aproximadamente 200 dias para as variedades Merlot e Cabernet Sauvignon. Em outro estudo, Leeuwen et al. (2004) verificaram que o ciclo dessas duas variedades, para a mesma região, foi de aproximadamente 175 dias. Para a variedade Merlot cultivada na Nova Zelândia, Friend \& Trougth (2007) observaram ciclo mais longo, com a colheita realizada entre o final de abril e o início de maio.

O comportamento vegetativo e produtivo das plantas apresentou variação entre os dois ciclos avaliados. Para a variedade Merlot, o número de ramos foi igual em ambos os ciclos; porém, no primeiro ciclo, foi superior para a Cabernet Sauvignon e inferior para a Sauvignon Blanc (Tabela 2). O comprimento dos ramos, em todas as variedades, foi maior no ciclo 2006/2007. Esse crescimento pode ser atribuído ao maior comprimento dos entrenós, pois o número de folhas por ramo não variou entre os ciclos. Isso indica que, no segundo ciclo, os carboidratos produzidos na fotossíntese foram armazenados nas estruturas vegetativas (ramos e raízes) em virtude do menor número de cachos. Essa situação ocasionou desequilíbrio entre as estruturas vegetativas e produtivas, o que resultou em vigor excessivo, confirmado pela elevada massa dos ramos podados.

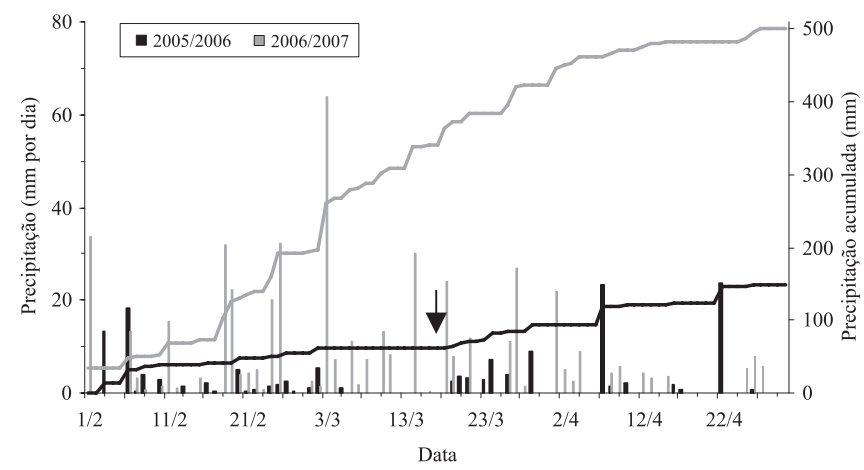

Figura 2. Precipitação diária (barras) e acumulada (linhas) durante o período de maturação das bagas da videira (Vitis vinifera L.) em São Joaquim, SC, nos ciclos 2005/2006 e $2006 / 2007$. A seta indica a data de irrigação do vinhedo durante o ciclo 2005/2006, em 20/3/2006.
Apesar de a área foliar não ter apresentado diferenças significativas, observou-se padrão homogêneo para todas as variedades, com tendência de maior desenvolvimento no segundo ciclo (Tabela 2). A área foliar por planta foi resultado da soma da área das folhas do ramo principal (cerca de 65\%) com a área das folhas distribuídas nas brotações laterais (feminelas), que representaram aproximadamente $35 \%$ do total. No ciclo 2005/2006, para a Sauvignon Blanc, a área foliar das plantas foi cerca de $15 \%$ inferior ao ciclo posterior. Para a Merlot, essa diferença foi de aproximadamente $10 \%$, enquanto para a Cabernet Sauvignon foi de cerca de 20\%. Myers et al. (2008) também observaram um padrão semelhante na proporção entre a área foliar dos ramos principais e das feminelas, ao estudar a variedade Sangiovese. Quanto à área foliar total por planta, há trabalhos que relatam valores semelhantes (Zoecklein et al., 2008); inferiores, de $4,6 \mathrm{~m}^{2}$ para Sauvignon Blanc (Petrie et al., 2003); e superiores, de $13,6 \mathrm{~m}^{2}$ para Sauvignon Blanc (Hunter, 2000), aos observados no presente trabalho.

O comportamento produtivo das plantas também apresentou variação expressiva, sendo o número e a massa dos cachos maior no primeiro ciclo. A produtividade no segundo ciclo foi de aproximadamente $75 \%$ do total colhido no ciclo 2005/2006 para as variedades Sauvignon Blanc e Merlot. Para a Cabernet Sauvignon, essa proporção foi de $67 \%$. O desequilíbrio no desenvolvimento vegetativo deste vinhedo durante o ciclo 2005/2006 pode ter ocasionado aumento da proporção de gemas inférteis, como descrito por Santos (2006), o que foi constatado pela redução no número de cachos por

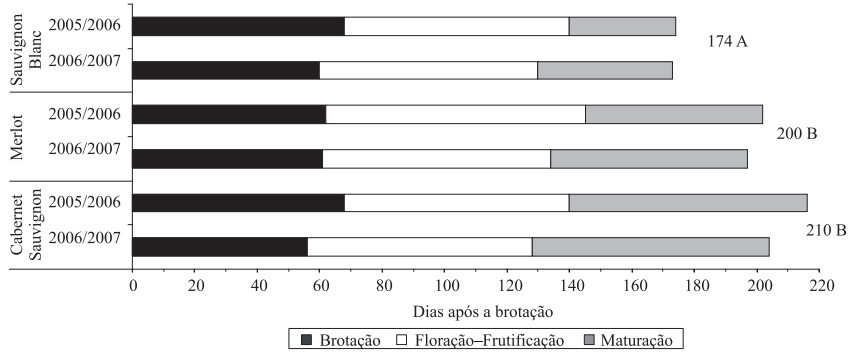

Figura 3. Estádios fenológicos das variedades Cabernet Sauvignon, Merlot e Sauvignon Blanc (Vitis vinifera L.) em São Joaquim, SC, durante os ciclos vegetativos 2005/2006 e 2006/2007. Brotação (setembro) e maturação (março e abril). 
planta e por ramo no ciclo 2006/2007. A menor massa média dos cachos e o menor rendimento por planta também foram ocasionados pelo desenvolvimento de doenças (podridões) nos cachos (ciclo 2006/2007), em decorrência do elevado volume e número de dias de chuva. O número de cachos por planta e o rendimento observados neste trabalho são inferiores aos descritos por outros autores (Petrie et al., 2003; Falcão et al., 2008; Myers et al., 2008; Silva et al., 2009).

O índice de Ravaz variou entre 1,4 e 2,1, para todas as variedades, o que indica desequilíbrio na distribuição dos fotoassimilados. Esse efeito gerou maior crescimento vegetativo como resultado da reduzida produção de uva por planta. De acordo com diversos autores, a relação produção/poda (índice de Ravaz) deve estar entre 5 e 10 (Kliewer \& Dokoozlian, 2005; Santos et al., 2006). Silva et al. (2009) observaram valores entre 3 e 6 para um vinhedo da variedade Syrah, sob as mesmas condições de cultivo. Esses resultados mostram que os fotoassimilados foram distribuídos preferencialmente para as estruturas de crescimento, o que favoreceu a continuidade do vigor vegetativo, conforme Santos et al. (2006).
A relação entre a área foliar e a produção diferiu significativamente entre os dois ciclos. No primeiro ciclo, os valores foram inferiores aos estimados para o ciclo posterior em virtude do menor rendimento por planta e da maior área foliar observada no segundo ciclo. Contudo, a relação entre a área foliar e a produção está muito acima dos limites considerados adequados por Kliewer \& Dokoozlian (2005), que variam entre 1,0 e 2,0 $\mathrm{m}^{2} \mathrm{~kg}^{-1}$ de uva. Em outro estudo com a variedade Merlot cultivada em São Joaquim, $\mathrm{SC}$, foi observado $4,5 \mathrm{~m}^{2} \mathrm{~kg}^{-1}$ de uva nas plantas que não receberam desponte dos ramos durante o ciclo 2006/2007 (Brighenti et al., 2010), o que indica que os fotossintatos foram mobilizados para os órgãos vegetativos.

Para todas as variedades, a uva colhida ao final do ciclo 2005/2006 apresentou teores mais elevados de sólidos solúveis totais, que se mantiveram acima de $23^{\circ}$ Brix (Tabela 2). No ciclo posterior, a maturação foi afetada pelas variáveis meteorológicas, o que resultou em teores próximos a $21^{\circ}$ Brix. Quanto à degradação dos ácidos orgânicos, não houve padrão entre os ciclos. Para a cultivar Sauvignon Blanc, os valores

Tabela 2. Comportamento vegetativo e produtivo, e composição da uva na colheita das variedades Cabernet Sauvignon, Merlot e Sauvignon Blanc (Vitis vinifera L.), cultivadas em São Joaquim, SC, nos ciclos 2005/2006 e 2006/2007.

\begin{tabular}{|c|c|c|c|c|c|c|c|c|c|c|c|c|}
\hline \multirow[t]{2}{*}{ Variável } & \multicolumn{4}{|c|}{ Sauvignon Blanc } & \multicolumn{4}{|c|}{ Merlot } & \multicolumn{4}{|c|}{ Cabernet Sauvignon } \\
\hline & \multicolumn{3}{|c|}{$2005 / 20062006 / 2007$} & & \multicolumn{3}{|c|}{$2005 / 20062006 / 2007^{(1)}$} & \multirow[t]{2}{*}{$\mathrm{CV}(\%)$} & \multicolumn{2}{|c|}{$2005 / 20062006 / 2007$} & \multicolumn{2}{|c|}{ (1) $\mathrm{CV}(\%)$} \\
\hline \multicolumn{10}{|l|}{ Comportamento vegetativo e produtivo } & & & \\
\hline Número de ramos por planta & 14,2 & 14,9 & $*$ & 13,1 & 7,8 & 7,5 & ns & 14,3 & 8,8 & 8,1 & $*$ & 13,8 \\
\hline Número de folhas por ramo & 35,9 & 32,3 & ns & 16,8 & 39,3 & 37,8 & ns & 14,3 & 36,1 & 32,5 & ns & 14,1 \\
\hline Comprimento do ramo principal $(\mathrm{cm})$ & 192,1 & 234,1 & $*$ & 16,4 & 212,4 & 322,8 & $*$ & 23,7 & 210,2 & 289,5 & $*$ & 26,7 \\
\hline Área foliar ( $\mathrm{m}^{2}$ por planta) & 9,99 & 12,13 & ns & 27,8 & 7,01 & 7,52 & ns & 22,8 & 5,51 & 6,66 & ns & 25,2 \\
\hline Área foliar do ramo principal ( $\mathrm{m}^{2}$ por planta) & 6,74 & 7,56 & ns & 19,1 & 4,88 & 4,58 & ns & 18,3 & 4,07 & 3,73 & ns & 20,6 \\
\hline Área foliar das brotações laterais ( $\mathrm{m}^{2}$ por planta) & 3,25 & 4,56 & ns & 42,4 & 2,12 & 2,94 & ns & 43,9 & 1,44 & 2,92 & $*$ & 50,7 \\
\hline Número de cachos por planta & 18,4 & 17,4 & ns & 17,9 & 12,0 & 10,1 & $*$ & 19,7 & 11,4 & 9,2 & $*$ & 28,2 \\
\hline Número de cachos por ramo & 1,3 & 1,2 & $*$ & 22,1 & 1,5 & 1,4 & $*$ & 22,8 & 1,3 & 1,1 & $*$ & 28,2 \\
\hline Massa média do cacho (g) & 147,5 & 116,4 & $*$ & 13,2 & 100,8 & 88,9 & $*$ & 16,5 & 108,9 & 90,9 & $*$ & 15,6 \\
\hline Produção (kg por planta) & 2,714 & 2,026 & $*$ & 31,5 & 1,209 & 0,898 & $*$ & 34,1 & 1,242 & 0,836 & $*$ & 30,0 \\
\hline AFT/P estimada $\left(\mathrm{cm}^{2} \mathrm{~g}^{-1} \text { de uva }\right)^{(2)}$ & 37 & 60 & - & - & 58 & 84 & - & - & 44 & 80 & - & - \\
\hline Massa da poda (kg por planta) & 1,238 & - & - & - & 0,980 & - & - & - & 0,925 & - & - & - \\
\hline Índice de Ravaz & 2,1 & - & - & - & 1,4 & - & - & - & 1,5 & - & - & - \\
\hline \multicolumn{13}{|l|}{ Composição das bagas } \\
\hline SST ( ${ }^{\circ}$ Brix $)$ & 23,8 & 21,0 & $*$ & 7,3 & 23,9 & 21,6 & $*$ & 5,5 & 23,3 & 21,2 & $*$ & 5,3 \\
\hline ATT (meq L-1) & 128,0 & 104,7 & $*$ & 11,8 & 87,3 & 106,7 & $*$ & 11,0 & 114,0 & 111,3 & ns & 5,3 \\
\hline $\mathrm{SST} / \mathrm{ATT}^{(3)}$ & 24,7 & 26,6 & ns & 5,3 & 36,4 & 27,0 & $*$ & 16,4 & 27,4 & 25,4 & ns & 6,6 \\
\hline $\mathrm{pH}$ & 2,86 & 3,01 & $*$ & 3,0 & 3,17 & 3,26 & $*$ & 1,6 & 3,14 & 3,31 & $*$ & 3,2 \\
\hline Coloração das sementes & 9,6 & 9,7 & ns & 6,0 & 9,8 & 9,5 & $*$ & 4,9 & 10,1 & 9,9 & $\mathrm{~ns}$ & 6,0 \\
\hline
\end{tabular}

${ }^{(1)}$ Diferença entre os ciclos para cada variedade. ${ }^{(2)} \mathrm{AFT} / \mathrm{P}$ estimada pela relação entre a área foliar total e a produção por planta. ${ }^{(3)} \mathrm{SST} / \mathrm{ATT}$ estimada pela relação entre o teor de SST ( ${ }^{\circ}$ Brix) e ATT (g por $100 \mathrm{~mL}$ ). ${ }^{\text {ns }}$ Não significativo. *Significativo pelo teste t de Student, a 5\% de probabilidade. (-) Dados não avaliados. 
foram superiores no primeiro ciclo. Já para a Merlot, a acidez total titulável foi maior nas bagas colhidas em 2006/2007. Para a Cabernet Sauvignon, essa variável não apresentou diferenças significativas no momento da colheita. Os valores de $\mathrm{pH}$ foram superiores no ciclo 2006/2007, em comparação ao ciclo anterior. Resultados semelhantes aos do presente trabalho foram obtidos por Falcão et al. (2008) e Brighenti et al. (2010), que também observaram níveis adequados de maturação fenólica para Cabernet Sauvignon e Merlot, em São Joaquim, SC.

A coloração das sementes foi semelhante entre os ciclos, com exceção da variedade Merlot. Os resultados observados para essa análise se encontram entre 9,5 e 10,1 e são compatíveis com os encontrados por Ristic \& Iland (2005). De acordo com estes autores, a coloração das sementes pode ser utilizada como um indicador complementar da maturação da uva.

Embora a precipitação tenha sido elevada durante a maturação no ciclo 2006/2007, a composição da uva foi adequada para a elaboração de vinhos. No momento da colheita, os índices de maturação foram considerados ótimos e comparáveis aos observados nas principais regiões de produção no mundo (Hunter et al., 2004; Leeuwen et al., 2004; Fidelibus et al., 2006; Friend \& Trought, 2007). Em geral, as variáveis analisadas nas bagas indicaram que a uva colhida no ciclo 2005/2006 apresentou maior teor de açúcares, menor acidez e melhor qualidade em comparação às colhidas no ciclo seguinte. A análise da coloração das sementes e da composição das bagas mostra que a uva atingiu maturação adiantada em ambos os anos estudados. No entanto, os índices de desenvolvimento indicam a necessidade de ajuste da carga de gemas para regular o equilíbrio entre o crescimento vegetativo e a produção, o que pode contribuir para melhorar a qualidade da uva de todas as variedades nessa região de cultivo.

\section{Conclusões}

1. Os estádios fenológicos das variedades Cabernet Sauvignon, Merlot e Sauvignon Blanc de videira estão de acordo com as variações normais entre os ciclos, com um desenvolvimento mais tardio em São Joaquim, $\mathrm{SC}$, em comparação às demais regiões de produção de uvas no Brasil.

2. A produção reduzida das plantas das variedades Cabernet Sauvignon, Merlot e Sauvignon Blanc induz o crescimento vegetativo excessivo e, portanto, há a necessidade de ajuste do manejo do dossel para promover o equilíbrio do vinhedo.

3. Há influência significativa da precipitação pluvial sobre a maturação da uva das variedades Cabernet Sauvignon, Merlot e Sauvignon Blanc.

4. As variedades Cabernet Sauvignon, Merlot e Sauvignon Blanc apresentam elevada qualidade da uva no momento da colheita, sendo adequadas ao cultivo em São Joaquim, Estado de Santa Catarina.

\section{Agradecimentos}

À Villa Francioni Agro Negócios S.A., por disponibilizar parte de seus vinhedos para a realização deste trabalho.

\section{Referências}

BLANCO-WARD, D.; QUEIJEIRO, J.M.G.; JONES, G.V. Spatial climate variability and viticulture in the Miño River Valley of Spain. Vitis, v.46, p.63-70, 2007.

BORGHEZAN, M.; GAVIOLI, O.; PIT, F.A.; SILVA, A.L. da. Modelos matemáticos para a estimativa da área foliar de variedades de videira à campo (Vitis vinifera L.). Ciência e Técnica Vitivinícola, v.25, p.1-7, 2010.

BRIGHENTI, A.F.; RUFATO, L.; KRETZSCHMAR, A.A.; MADEIRA, F.C. Desponte dos ramos da videira e seu efeito na qualidade dos frutos de 'Merlot' sobre porta-enxertos 'Paulsen 1103' e 'Couderc 3309'. Revista Brasileira de Fruticultura, v.32, p.19-26, 2010.

CLOETE, H.; ARCHER, E.; HUNTER, J.J. Shoot heterogeneity effects on Shiraz/Richter 99 grapevines. I. Vegetative growth. South African Journal of Enology and Viticulture, v.27, p.68-75, 2006.

ESTEVES, M.A.; ORGAZ, M.D.M. The influence of climatic variability on the quality of wine. International Journal of Biometeorology, v.45, p.13-21, 2001.

FALCÃO, L.D.; CHAVES, E.S.; BURIN, V.M.; FALCÃO, A.P.; GRIS, E.F.; BONIN, V.; BORDIGNON-LUIZ, M.T. Maturity of Cabernet Sauvignon berries from grapevines grown with two different training systems in a new grape growing region in Brazil. Ciencia e Investigación Agraria, v.35, p.271-282, 2008.

FIDELIBUS, M.W.; CHRISTENSEN, L.P.; KATAYAMA, D.G.; VERDENAL, P.T. Yield components and fruit composition of six 'Cabernet Sauvignon' grapevine selections in the Central San Joaquin Valley, California. Journal of the American Pomological Society, v.60, p.32-36, 2006.

FRIEND, A.P.; TROUGHT, M.C.T. Delayed winter spur-pruning in New Zealand can alter yield components of Merlot grapevines. Australian Journal of Grape and Wine Research, v.13, p.157-164, 2007. 
GIRONA, J.; MARSAL, J.; MATA, M.; CAMPO, J.; BASILE, B. Phenological sensitivity of berry growth and composition of Tempranillo grapevines (Vitis vinifera L.) to water stress. Australian Journal of Grape and Wine Research, v.15, p.268-277, 2009.

HUNTER, J.J. Implications of seasonal canopy management and growth compensation in grapevine. South African Journal of Enology and Viticulture, v.21, p.81-91, 2000.

HUNTER, J.J.; VOLSCHENK, C.G.; MARAIS, J.; FOUCHÉ, G.W. Composition of Sauvignon blanc grapes as affected by pre-véraison canopy manipulation and ripeness level. South African Journal of Enology and Viticulture, v.25, p.13-18, 2004.

JONES, G.V.; DAVIS, R.E. Climate influences on grapevine phenology, grape composition, and wine production and quality for Bordeaux, France. American Journal of Enology and Viticulture, v.51, p.249-261, 2000.

KLIEWER, W.M.; DOKOOZLIAN, N.K. Leaf area/crop weight ratios of grapevines: influence on fruit composition and wine quality. American Journal of Enology and Viticulture, v.56, p.170-181, 2005.

LEEUWEN, C.; FRIANT, P.; CHONÉ, X.; TRÉGOAT, O.; KOUNDOURAS, S.; DUBOURDIEU, D. The influence of climate, soil and cultivar on terroir. American Journal of Enology and Viticulture, v.55, p.207-217, 2004.

LORENZ, D.H.; EICHHORN, K.W.; BLEIHOLDER, H.; KLOSE, R.; MEIER, U.; WEBER, E. Phenological growth stages of the grapevine (Vitis vinifera L. ssp. vinifera) - codes and descriptions according to the extended BBHC scale. Australian Journal of Grape and Wine Research, v.1, p.100-103, 1995.

MIELE, A.; RIZZON, L.A.; ZANUS, M.C. Discrimination of Brazilian red wines according to the viticultural region, varietal, and winery origin. Ciência e Tecnologia de Alimentos, v.30, p.268-275, 2010.

MOTA, C.S.; AMARANTE, C.V.T.; SANTOS, H.P. dos; ZANARDI, O.Z. Comportamento vegetativo e produtivo de videiras 'cabernet sauvignon' cultivadas sob cobertura plástica. Revista Brasileira de Fruticultura, v.30, p.148-153, 2008.

MYERS, J.K; WOLPERT, J.A.; HOWELL, S. Effect of shoot number on the leaf area and crop weight relationship of young
Sangiovese grapevines. American Journal of Enology and Viticulture, v.59, p.422-424, 2008.

ORGANIZATION INTERNATIONALE DE LA VIGNE ET DU VIN. Compendium of international methods of wine and must analysis. Paris: OIV, 2009. v.1, 419p.

PETRIE, P.R.; TROUGHT, M.C.T.; HOWELL, G.S.; BUCHAN, G.D. The effect of leaf removal and canopy height on whole-vine gas exchange and fruit development of Vitis vinifera L. Sauvignon blanc. Functional Plant Biology, v.30, p.711-717, 2003.

RISTIC, R.; ILAND, P.G. Relationships between seed and berry development of Vitis vinifera L. cv Shiraz: developmental changes in seed morphology and phenolic composition. Australian Journal of Grape and Wine Research, v.11, p.43-58, 2005.

RIZZON, L.A.; MIELE, A. Avaliação da cv. Merlot para elaboração de vinho tinto. Ciência e Tecnologia de Alimentos, v.23, p.156-161, 2003

SANTOS, H.P. dos. Aspectos ecofisiológicos na condução da videira e sua influência na produtividade do vinhedo e na qualidade dos vinhos. Bento Gonçalves: Embrapa Uva e Vinho, 2006. 9p. (Embrapa Uva e Vinho. Comunicado técnico, 71).

SANTOS, H.G. dos; JACOMINE, P.K.T.; ANJOS, L.H.C. dos; OLIVEIRA, V.A. de; OLIVEIRA, J.B. de; COELHO, M.R.; LUMBRERAS, J.F.; CUNHA, T.J.F. (Ed.). Sistema brasileiro de classificação de solos. 2.ed. Rio de Janeiro: Embrapa Solos, 2006. $306 \mathrm{p}$.

SILVA, L.C. da; RUFATO, L.; KRETZSCHMAR, A.A.; MARCON FILHO, J.L. Raleio de cachos em vinhedos de altitude e qualidade do vinho da cultivar Syrah. Pesquisa Agropecuária Brasileira, v.44, p.148-154, 2009.

STEEL, R.G.D.; TORRIE, J.H.; DICKEY, D.A. Principles and procedures of statistics: a biometrical approach. $3^{\text {rd }}$ ed. New York: McGraw-Hill, 1997. 666p.

TONIETTO, J.; CARBONNEAU, A. A multicriteria climatic classification system for grape-growing regions worldwide. Agricultural and Forest Meteorology, v.124, p.81-97, 2004.

ZOECKLEIN, B.W.; WOLF, T.K.; PÉLANNE, L.; MILLER, M.K.; BIRKENMAIER, S.S. Effect of vertical shoot-positioned, smart-dyson, and geneva double-curtain training systems on Viognier grape and wine composition. American Journal of Enology and Viticulture, v.59, p.11-21, 2008.

Recebido em 3 de janeiro de 2011 e aprovado em 30 de março de 2011 